and note that, as such, many of its symptoms resemble those of post-traumatic stress disorder . . ." (Prigerson et al, 1999; p. 67). In fact, we refer to the syndrome as traumatic grief in recognition of the fact that we repeatedly find symptoms of traumatic distress (symptoms in criterion B) to form a unidimensional factor with symptoms of separation distress (grief). If anything, we believe our research has drawn attention to the phenomenological overlap between pathological grief and post-traumatic stress symptomatology.

Nevertheless, we do not consider the overlap to be complete. Criteria for PTSD do not include the core symptoms of separation distress such as yearning, longing, or searching for the deceased, or excessive loneliness resulting from the loss. The unbidden, intrusive thoughts and longings are related to a wish to be reunited with the deceased, and it is the absence of the person that is distressing rather than fears that the horrifying event will be reexperienced (Raphael \& Martinek, 1997). Re-experiencing the presence of the deceased more typically proves to be a comfort and not a source of distress (Jacobs, 1993; Raphael \& Martinek, 1997).

Whereas avoidance is a cardinal feature of PTSD, as our results indicated, the avoidance item had low specificity, an extremely low item-total correlation $(r=0.01)$, and Cronbach's alpha for the criteria set improved with its deletion. The poor performance and low mean levels of the avoidance item were also found in Spooren et al's (1999) study of parents several years after their child's death. Similarly, item response theory analyses we recently conducted on a community sample of 76 widows and widowers revealed the avoidance item to yield the least amount of information for distinguishing between cases and non-cases of traumatic grief. Contrary to Fox's clinical observations, longitudinal data presented by Horowitz et al (1997) indicate that the frequency of bereaved subjects in his sample who avoided thoughts of the deceased dropped from $52.9 \%$ at 6 months post-loss to $12.9 \%$ at 14 months post-loss (when its specificity for predicting complicated grief was only 0.26 ), suggesting that avoidance phenomena are more likely to dissipate over time than they are to have a delayed presentation. Rather than avoidance, the more salient behaviour is one of searching for reminders of the deceased. In traumatic grief the hypervigilance relates to scanning the environment for cues of the deceased, rather than to fears that the traumatic event will be re-experienced. While PTSD criteria in DSM-IV include sleep disturbances, in an electroencephalographic sleep study, the sleep architecture revealed no evidence of hyperaroused sleep among subjects with syndromal-level traumatic grief (McDermott et $a l, 1997)$. The evidence appears to be mounting that avoidance per se (as opposed to the highly informative dissociative symptoms such as numbness) is not an efficient marker for traumatic grief, and hyperarousal manifests itself in a distinctive way among those with traumatic grief.

In terms of overall diagnostic agreement, in a sample of 76 young adult friends who had been exposed to a friend's suicide, we found that three of the seven $(43 \%)$ who met SCID criteria (Spizzer et al, 1990) for PTSD did not meet our criteria for caseness of traumatic grief, and 12 of the $16(75 \%)$ who met our criteria for traumatic grief did not meet criteria for PTSD (PTSD-traumatic grief kappa=0.27). Thus, although there is substantial overlap between symptoms of traumatic grief and those of PTSD, these disorders do not appear isomorphic, and traumatic grief may prove to be a unique type of stress response syndrome.

Classifying pathological grief responses as adjustment disorders appears an inadequate alternative. Adjustment disorders do not include the specific clinical features of traumatic grief described above. The clinical description of adjustment disorder is imprecise, and would indicate little more than that a widowed person was having difficulty adjusting to the loss. The stipulation in DSM-IV (American Psychiatric Association, 1994) that adjustment disorder "must resolve within 6 months of the termination of the stressor" runs counter to the longer duration Fox et al recommend and would exclude the large number of bereaved individuals with symptoms of traumatic grief beyond 6 months. Lastly, criterion D for adjustment disorder explicitly states that the symptoms cannot be a consequence of bereavement. For these reasons, adjustment disorder does not appear to be appropriate or clinically useful for bereaved individuals who suffer from symptoms of traumatic grief, particularly, as it is defined in DSM-IV.

While we and other members of the consensus panel agree that two months post-loss seems premature to make a diagnosis of traumatic grief, we were obliged to report the recommendations of the consensus panel. We await the analysis of longitudinal data collected on the proposed criteria among a more representative sample of widowed elders before drawing firmer conclusions about the preferred timing of the onset/duration of symptoms that should be required for a diagnosis of traumatic grief. We consider this the first step of many towards refining a clinically useful criteria set for this disorder.

American Psychiatric Associntion (1994) Diognostic and Statistical Monual of Mental Disorders (4th edn) (DSM-N). Washington. DC: APA

Horowitz, M. J., Siegel, B., Holen, A., et of (1997) Criteria for complicated grief disorder. Americon fournol of Psychiatry, 154, 905-910.

Jacoba, S. C. (1993) Pathological Grief. New York: American Psychiatric Press.

McDermot, O., Prigerson, H. G., Houck, P. R., et ol (1997) Sleep in the wake of complicated grief. Biological Psychiotry. 41. 710-716.

Prigerson, H. G., Shear, M. K., becobs, S. C., *e el (1999) Consensus criteria for traumatic grief. A preliminary empirical test. British joumal of Psychiotry, 174, 67-73.

Raphad, B. Martinek, N. (I9M) Assessing traumatic bereavement and PTSD. In Assessing Psychological Trouma and PTSD (eds ). P. Wilson \& T. M. Keane), pp. 373-395. New York: Guilford Press.

Spitzer, R. L., Millams, J. B.W. Gibbon, M., et al (19\%0) Structured Clinical Interview for DSM-III-R Non-patient Edition (SCID-NP, version 1.0). Washington. DC: American Psychiatric Press.

Spooren, D. J., Henderisk, M. \& Jannes, C. (1999) A retrospective study of parents bereaved by a child in a traffic accident. Omego, in press.

H. G. Prigerson Yale University School of Medicine, 34 Park Street, New Haven, CT 06519, USA

S. C. Jacobs, R. A. Rosenheck,

P. K. Maciejewsid Northeast Program

Evaluation Center, VA Medical Center (182),

Campbell Avenue, West Haven, CT 06516, USA

\section{Behavioural changes and psychiatric symptoms in Alzheimer's disease}

Sir: In their article on behavioural changes and psychiatric symptoms in Alzheimer's disease, Hope et al (1999) state that from their findings "it is worthwhile considering a trial withdrawal of any medication which is prescribed for psychiatric or behavioural changes in dementia". They also state that "if a trial without treatment was instituted ... then $25 \%$ of them would not have the index problem any more". These statements, along with other comments in the paper, suggest that all patients with physical 
aggression (62\% as defined in the study) should be on medication.

The evidence that drug treatments have major benefit in the management of such behaviour remains less than convincing (Schneider et al 1990, Schneider \& Sobin, 1991) and side-effects of most drugs involved can be disabling. Effects such as lethargy, akathisia and akinesia can develop insidiously, and are often not recognised, being attributed to the disease process rather than to the drug. Treatment is often, perhaps usually, given without proper informed consent (Jackson \& McGrath, 1996).

Physical aggression in people with dementia is not simply due to the disease process, but is affected by many factors including the expectations, training and tolerance of carers; the care environment; physical illness or discomfort; and the personality of the aggressor. These problems must be taken into account, and modified where possible.

A decision to manage physical aggression using regular drug treatment should not be taken lightly. Rather than "considering a trial withdrawal every 8 months", whether or not to continue use of medication should be continually reassessed. It is important to look at alternative managements at all times, and consider the risk: benefit ratio, that is, the not insignificant risk of side-effects compared with any perceived benefit. A Scottish guideline group (Scottish Intercollegiate Guidelines Network, 1998) suggests that "neuroleptics should only be considered for patients with serious problems, in particular psychotic symptoms, or in the presence of serious distress or danger from behaviour disturbance". This document also discusses other aspects of management.

Hope et al's article is a welcome addition in its description of the behavioural and psychiatric problems associated with dementia, particularly in discussing its natural history. However, their "clinical implications" on medication use are not supported with their findings.

Hope, T, Keene, J., Fuiturn, C. G., ot of (Isse) Natural history of behavioural changes and psychiatric symptoms in Alzheimer's disease. A longitudinal study. British fournal of Pychiatry, 174, 39-44.

Jaclaon, G. A. \& Mccrath, A. M. (I9\%). Consent to treatment. British journol of Psychiotry, 169, 382-383.

Schmoider, L. S., Pollock, V. E. \& Lmeas, S. A. (19\%0) A metanalysis of controlled trials of neuroleptic treatment in dementia. fournol of the Americon Geriatric Society, 38, 553-563.

- Sobin, P. B. (1991) Non-neuroleptic medication in the management of agitation in Alzheimer's disease and other dementia: a selective review. Internotional joumal of Geriatric Psychiatry, 6, 691-708.

Scottish Intercolleglate Culdalines Network (SICN) (19s9) Interventions in the Manogement of Behovioural and Psychological Aspects of Dementia. Edinburgh: SIGN.

G. A. Jackson Leverndale Hospital,

510 Crookston Road, Glasgow G537TU

Author's reply: We welcome the letter from Dr Jackson. We did not intend in our paper to make any presumptions about the efficacy or otherwise of medical treatment for aggressive behaviour. This is an issue outside the remit of that paper. The point we wanted to stress is that since the natural history (untreated) of most behavioural problems in dementia is for them to remit spontaneously, it is important that patients who are given medication are not left on that medication without a trial of withdrawal. Our purpose, like that of Dr Jackson, in discussing the clinical implications of our findings was to stress the point that a patient should not be left on medication without review.

T. Hope Ethox, Institute of Health Sciences, Old Road, Headington, Oxford OX37LF

\section{Detecting vascular pathology in vascular dementia}

Sir: In their study of older people on the Camberwell Dementia Case Register, Holmes et al (1999) highlight the influence of prevalence rates and mixed pathology on the validity of current diagnostic systems. However, it is possible that both clinical and neuropathological classificatory systems have underestimated the presence of vascular pathology.

It is unclear as to precisely how the location of infarcts influenced quantitative assessment of vascular pathology in the study, as it is known that strategic infarcts in frontal/subcortical structures may be associated with cognitive impairment, but are not traditionally seen as implicated in the development of dementia (Rao \& Howard, 1998). It is also surprising that the neuropathological assessment of cerebrovascular disease was confined to infarcts, as Neurological and Communicative Disorders and Stroke and the Alzheimer's Disease and Related Disorders Association (NINDS-AIREN) criteria for vascular dementia also allow the presence of extensive periventricular white matter lesions (Roman et al, 1993).

Although the presence of 'pure' vascular pathology comprised less than $10 \%$ of the total sample, it is striking that four people with infarcts alone at post-mortem were mis-classified as non-probable vascular dementia according to NINDS-AIREN criteria. Three of these had no clinical evidence of focal neurological signs; such 'silent' strokes are known to be associated with cognitive impairment (Price et al, 1997). Lastly, given the high prevalence of mixed pathology in the general population, it is suggested that the incorporation of mixed pathology into diagnostic criteria for both Alzheimer's disease and vascular dementia may be needed for research and treatment. The problem with mixed pathology is that it represents a nosologically heterogeneous group of disease processes, further compounded by the inextricable relationship between vascular risk factors and Alzheimer's disease (Prince et al, 1994). The way forward may be to improve the sensitivity of current diagnostic instruments by allowing a broader definition of both cerebrovascular disease and cognitive impairment, taking into account the wide range of aetiologies encountered in vascular dementia. This will not only encompass a larger group with 'pure' cerebrovascular lesions, but also serve to treat a distinct disorder that is still unduly influenced by the neuropsychological characterisation of Alzheimer's disease.

Holmes, C., Calms, N., Lantos, P., ot al (IS99) Validity of current clinical criteria for Alzheimer's disease. vascular dementia and dementia with Lewy bodies. British journal of Psychiatry, 174, 45-50.

Price, T. R., Manolio, T. A., Kronmal, R. A., ete of (IS)T Silent brain infarction on magnetic resonance imaging and neurological abnormatities in communitydwelling older adults. The Cardiovascular Health Study. CHS Collaborative Research Group. Stroke, 28. $1158-1164$

Prince, M, Cullen, M. \& Mann, A. (I9s4) Risk factors for Alzheimer's disease and dementia: a case-control study based on the MRC elderty typer tension trial. Neurologx 44, 97-104.

Rao, R. \& Howard, R. (1993) Vascular dementia Dead or alive? International foumal of Geriotric Psychiatry 13. 277-284.

Roman, G. C., Beamichi, T. K., Ertdnjunked, T, $\propto$ of (IS93) Vascular dementia: diagnosis criteria for research studies: Report of the NINDS-AIREN International workshop. Neurology, 43, 250-260.

R. Rao Job Ward, Thomas Guy House,

Guy's Hospital, London SEI 9RT 